\title{
Identification of Immunogenic Epitopes That Permit the Detection of Antigen-Specific T Cell Reponses to Coxsackievirus B3
}

Troy Wesson ${ }^{1}$, Adeeba Dhalech ${ }^{2}$, Christopher M. Robinson ${ }^{2}$

${ }^{1}$ Indiana University School of Medicine, ${ }^{2}$ Department of Microbiology and Immunology, Indiana University School of Medicine

\section{Background and Hypothesis:}

Coxsackievirus B3 (CVB3) is a non-enveloped RNA virus from the Picornaviridae family and is a primary cause of viral myocarditis in the United States. Approximately $5 \%$ of all symptomatic CVB3 infections are fatal. Therefore, there is a need to identify the mechanism(s) that regulate a protective immune response to CVB3. However, viral epitopes that stimulate $\mathrm{T}$ cell responses to CVB3 remain poorly characterized. To this end, we used a mouse model of CVB3 infection to identify the viral immunogenic CD8 T cell epitopes. We hypothesized that isolated antigenexperienced CD8 T cells from infected mice would be stimulated in the presence of predicted viral epitopes, confirming CVB3-specific T cells.

\section{Experimental Design:}

To identify novel CD8 T cell epitopes, predicted 9-mer MHC binding peptides from the CVB3Nancy polyprotein were identified using the Immune Epitope Database (IEDB) analysis resource consensus tool. The top ten predicted peptides were synthesized for our assays. Splenocytes from CVB3-infected male and female IFNAR -/- mice were stimulated with each peptide in the presence of brefeldin A for 6 hours at $37^{\circ} \mathrm{C}$. Following stimulation, cells were surfaced stained with antibodies specific for antigen-experienced CD8 T cells. Next, we performed intracellular staining for IFN-gamma. Cells were analyzed using flow cytometry. Candidate epitopes were identified as having results $\geq 2$ standard deviations over the control.

\section{Results:}

Thus far, our analysis has revealed responses to three novel CD8 T cell epitopes within the peptide library, including the viral epitopes within VP1 protein and the RNA-dependent RNA polymerase.

\section{Conclusion and Potential Impact}

Overall, these data provide an advancement in CVB3 immunology. Further, these data generate new tools like MHC-tetramers to track endogenous T cell responses to CVB3 infection. 
